\section{Compromiso óseo en mastocitosis sistémica: una asociación frecuente, pero muchas veces inadvertida. Casos clínicos}

\author{
PABLO FLORENZANO ${ }^{1}$, VERÓNICA MEZZANO², \\ MARCELA LE-BERT ${ }^{3}$, GILBERTO GONZÁLEZ ${ }^{1}$
}

\section{Bone involvement in systemic mastocytosis. Report of two cases}

Systemic mastocytosis (SM) is characterized by pathologic expansion and activation of mast cells. The main clinical manifestations of SM include skin involvement, gastrointestinal symptoms and anaphylaxis due to the release of its mediators. Thirty percent of patients with SM have a low bone mass and 20\% fractures. At the same time, SM affects $10 \%$ of male patients with idiopathic osteoporosis. Measuring serum tryptase is essential for the screening of MS. We report two cases of SM with bone involvement. A 25-year-old woman with prior diagnosis of $S M$, based on skin involvement, flushing, high serum tryptase and compatible bone marrow (BM) biopsy and genetic study. Low bone mass was diagnosed and treatment was started with calcium and vitamin D plus oral bisphosphonates with adequate response. A 47 years old man who presented with multiple osteoporotic vertebral fractures and low bone mass. Treatment with vitamin $D$ and alendronate was started, but the patient developed new vertebral fractures. The study was extended with measurement of serum tryptase that was elevated. Diagnosis of SM was confirmed with BM biopsy and the patient was referred to hematology for specific care. These cases emphasize the importance of bone assessment in SM, as well as the need to rule out SM in patients with osteoporosis and no evident cause.

(Rev Med Chile 2016; 144: 401-405)

Key words: Fractures; Mastocytosis; Osteoporosis; Systemic.

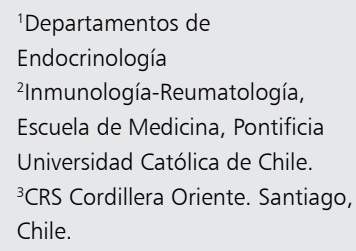

Recibido el 5 de noviembre de 2015, aceptado el 12 de enero de 2016.

\section{Correspondencia a:}

Dr. Gilberto González V.

Departamento de Endocrinología,

Escuela de Medicina, Pontificia

Universidad Católica de Chile.

Lira 85. $5^{\circ}$ Piso, Santiago, Chile.

Código Postal 8330023

56-2-23543095

ggonzale@med.puc.cl

\section{L} a mastocitosis sistémica (MS) se caracteriza por la proliferación y activación patológica de mastocitos, los que se encuentran en distintos tejidos y liberan mediadores sistémicos tales como histamina, proteasas, heparina, prostaglandinas, leucotrienos y citoquinas ${ }^{1-2}$. Derivado de lo anterior, la presentación clínica de la MS es variable e inespecífica. El compromiso cutáneo y reacciones anafilácticas son la presentación clínica más conocida de MS. Sin embargo, la MS también puede tener otras formas, menos conocidas de presentación, como por ejemplo osteoporosis grave ${ }^{3}$.
A nivel óseo la MS puede manifestarse por osteoporosis, osteoesclerosis o lesiones focales mixtas, siendo la osteoporosis la manifestación más frecuentemente objetivada. Liberación de citoquinas y mediadores inflamatorios determina aumento del remodelamiento óseo con predominio habitualmente de la resorción sobre la formación ósea. Esto lleva a una pérdida significativa de masa ósea ${ }^{3}$. Entre 18-31\% de los pacientes con MS tendrán osteoporosis y 1 de cada 5 pacientes tendrá una fractura vertebral al momento del diagnóstico de $\mathrm{MS}^{4-6}$. 
Nuestro objetivo es enfatizar la importancia del compromiso óseo en MS y viceversa, la necesidad de considerar también a la MS como causa de osteoporosis en algunas circunstancias. Para este efecto se comunican dos casos de MS con compromiso óseo destacando aspectos de su diagnóstico y manejo junto con una revisión actualizada de esta asociación, bien conocida, pero muchas veces inadvertida.

\section{Casos clínicos}

\section{Caso 1}

Mujer de 25 años, sin antecedentes previos de importancia. A los 23 años presentó lesiones cutáneas eritematosas solevantadas en extremidades inferiores y tronco con signo de Darier positivo, asociadas a lipotimia y flushing al exponerse a calor. Niega reacciones urticariales y episodios de anafilaxia. Biopsia cutánea confirmó urticaria pigmentosa. Se complementó estudio con triptasa sérica que resultó en $40 \mathrm{ng} / \mathrm{ml}$ (valor normal $(\mathrm{vn}) \leq 11,4 \mathrm{ng} / \mathrm{ml})$. Biopsia de médula ósea fue compatible con MS. Se realizó estudio genético objetivándose mutación de cKIT D816V (+). Se diagnosticó MS indolente y se inició tratamiento con cromoglicato de sodio.

Se buscó dirigidamente el compromiso óseo, sin historia de dolor óseo, litiasis ni fracturas. Consumía 2 unidades lácteas/día. Menarquia a los 12 años y tenía ciclos menstruales regulares. Era nuligesta y no usaba anticonceptivos hormonales. Se solicitó densitometría ósea (DXA) que mostró DMO en L2-L4 0,880 g/ $\mathrm{cm}^{2}$ (Z score $-2,3$ ) y en cuello femoral de $0,719 \mathrm{~g} / \mathrm{cm}^{2}$ ( $Z$ score $-2,1)$. Se diagnosticó baja masa ósea secundaria a MS. Se optimizó aporte de calcio y vitamina D y se indicó Ibandronato $150 \mathrm{mg} / \mathrm{mensual}$ oral. Se mantuvo este manejo por dos años, tras lo cual fue estudiada por primera vez en nuestro centro. Se actualizó estudio: perfil bioquímico normal, creatinina $0,78 \mathrm{mg} / \mathrm{dL}$, función tiroidea normal, 25OHD 32 ng/ml (vn: $\geq 20$ ), Carboxitelopéptido sérico (CTx) 0,147 ng/ml (vn: 0,025-0,573 ng/ $\mathrm{ml}$ ). DXA de control mostró aumento de DMO de 7,2\% en L2-L4 y 8,3\% en caderas. La paciente refirió deseo de fertilidad por lo que se decidió la suspensión de Ibandronato y seguimiento con DXA y marcadores de resorción óseo (CTx).
Caso 2

Hombre de 47 años que consultó por fracturas vertebrales múltiples sin traumatismo identificable (L1, L2 y L4). Se solicitó DXA que mostró DMO en L2-L4 de $0,896 \mathrm{~g} / \mathrm{cm}^{2}$ (Z score-2,6) y de 0,795 $\mathrm{g} / \mathrm{cm}^{2}(Z$ score- 1,5$)$ en cuello femoral. En la anamnesis sólo destacó tabaquismo 10 cigarrillos/día con ingesta de 2 unidades lácteos/día. El estudio de causas secundarias fue negativo (Tabla 1). Se planteó Osteoporosis Idiopática del Varón. Se manejó con optimización de aportes de lácteos y vitamina $\mathrm{D}$ más alendronato $70 \mathrm{mg}$ oral semanal. El control con DXA al año de tratamiento mostró mantención de DMO en columna lumbar con aumento de $6 \%$ en cuello femoral. Tres años después se evidenció en resonancia magnética nuevas fracturas en L4 y L5. Se repitió estudio de causas secundaria, agregando esta vez medición de triptasa sérica que resultó en 42,6 ng/ml. El

Tabla 1. Estudio complementario caso 2

\begin{tabular}{|lcc|}
\hline Examen & $\begin{array}{c}\text { Rango de } \\
\text { referencia }\end{array}$ & Resultado \\
\hline Hematocrito & $41-53 \%$ & 45,4 \\
\hline Leucocitos & $4,5-111.000 \mathrm{cel} / \mathrm{mm}^{3}$ & 6,4 \\
\hline VHS & $1-14 \mathrm{~mm} / \mathrm{h}$ & 3 \\
\hline Testosterona total & $132-892 \mathrm{ng} / \mathrm{dL}$ & 461 \\
\hline LH & $1,5-9,3 \mathrm{mUl} / \mathrm{ml}$ & 4 \\
Calcio & $8,5-10,5 \mathrm{mg} / \mathrm{dl}$ & 9,8 \\
\hline Fósforo & $2,6-4,5 \mathrm{mg} / \mathrm{dl}$ & 3,1 \\
\hline Albúmina & $3,5-5 \mathrm{~g} / \mathrm{dL}$ & 4,8 \\
\hline TSH & $0,3-4,2 \mathrm{mUl} / \mathrm{mL}$ & 1,61 \\
Creatinina & $0,7-1,2 \mathrm{mg} / \mathrm{dL}$ & 0,8 \\
\hline 25 OH VIT D & $20-100 \mathrm{ng} / \mathrm{ml}$ & 18,6 \\
\hline PTH & $15-65 \mathrm{pg} / \mathrm{mL}$ & 29 \\
\hline Calcio urinario & $<300 \mathrm{mg} / 24 \mathrm{~h}$ & $259 \mathrm{mg} /$ \\
\hline $24 \mathrm{~h}$ & & $24 \mathrm{~h}$ \\
\hline Creatinina urinaria & $0,63-2,5 \mathrm{~g} / 24 \mathrm{~h}$ & 1,6 \\
$24 \mathrm{~h}$ & & $133 \mathrm{U} / \mathrm{L}$ \\
\hline Fosfatasas alcalinas & $45-115 \mathrm{UL}$ & 0,583 \\
\hline Ctx sérico & $(0,016-0,584)$ & $<, 020 /$ \\
\hline Cortisol salival & $<0,1 \mathrm{ug} / \mathrm{dL}$ & $\mathrm{Normal}$ \\
nocturno & & \\
\hline Electroforesis de & & \\
proteínas & & \\
\hline
\end{tabular}


paciente no presentó síntomas ni signos clínicos de MS. Se confirmó diagnóstico con biopsia de MO. Se diagnosticó osteoporosis secundaria a MS, se inició Denosumab 60 mg s.c. cada 6 meses y se derivó a Hematología e Inmunología para manejo de su MS.

\section{Discusión}

Los mastocitos son células residentes de distintos tejidos como la piel, mucosa respiratoria y gastrointestinal. Son responsables de los síntomas asociados a reacciones alérgicas como el prurito, broncoconstricción y vasodilatación debido a la liberación de mediadores como histamina, proteasas e. g. triptasa, heparina, prostaglandinas, leucotrienos y citoquinas. Fisiológicamente, la liberación de estos mediadores se produce al activarse el receptor de IgE tras la exposición del alérgeno al mastocito. En la MS existe un mayor número y actividad de mastocitos, pudiendo degranularse con estímulos inespecíficos como calor, grataje, algunos alimentos o medicamentos. Los mastocitos provienen de precursores hematopoyéticos que maduran al ser activados por el SCF (Stem Cell Factor), uniéndose a su receptor de membrana, denominado c-KIT. Hoy sabemos que la causa de la MS obedece a una mutación activante constitutiva del gen que codifica para el receptor cKIT.
La Organización Mundial de la Salud propone clasificar las MS según su agresividad y compromiso funcional de los órganos comprometidos en Indolentes, Asociadas a enfermedad hematológica, Agresiva y Leucemias/sarcomas mastocitarios ${ }^{7}$. Clínicamente la MS puede presentar: compromiso muco-cutáneo con urticaria pigmentosa (máculo-pápulas eritemato-pigmentadas, monoformas), urticaria crónica, angioedema o flushing; síntomas gastrointestinales como dolor cólico, náuseas, vómitos y diarrea; síntomas respiratorios obstructivos y finalmente anafilaxia, espontánea o asociada a picaduras de himnópteras. Al examen puede objetivarse el signo de Darier (reacción urticarial en los 5 minutos post grataje de una lesión). Ante la sospecha diagnóstica por lesiones cutáneas se recomienda proceder con una biopsia buscando un infiltrado mastocitario en la dermis superior ${ }^{1}$. En caso de ausencia de manifestaciones cutáneas se debe solicitar triptasa sérica total como test de tamizaje (Figura 1$)^{1,3,8}$. Los niveles de triptasa reflejan la masa y nivel de activación de mastocitos. Si se encuentra elevada el paciente debe ser derivado para evaluación por especialista. La confirmación de MS se realiza mediante biopsia de $\mathrm{MO}$ con estudio histoquímico ${ }^{1}$. Finalmente, la mutación de cKIT es detectable hasta en un 90\% de los adultos con MS, transformándose en un complemento para el diagnóstico de $\mathrm{MS}^{2}$. Los mecanismos del compromiso óseo en la MS se han relacionado a la liberación de TNF- $\alpha$, IL-1,

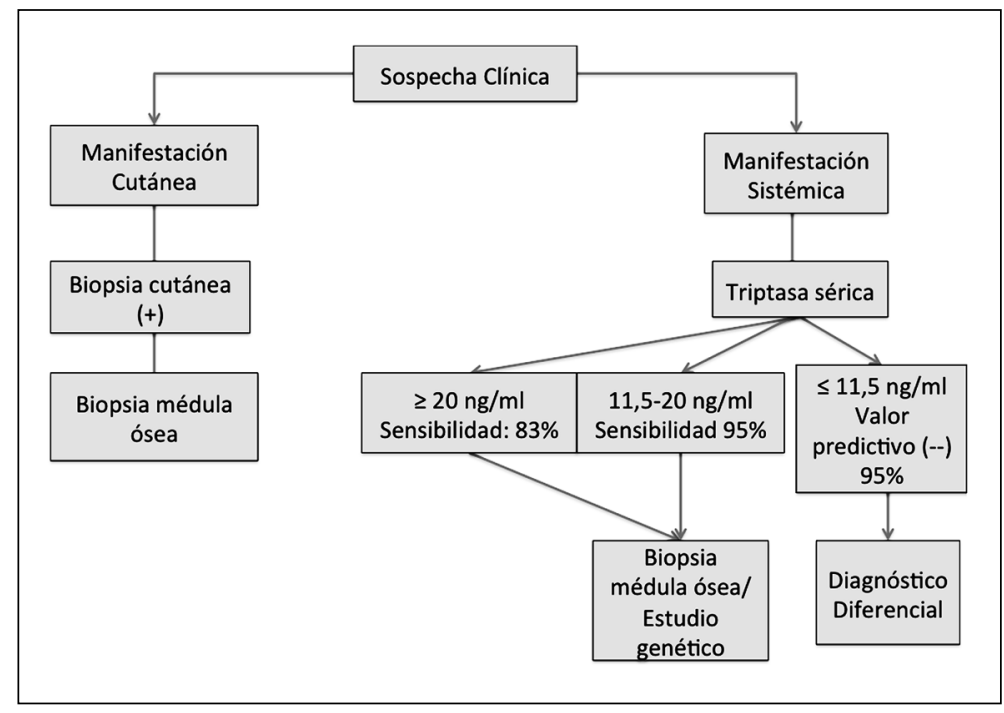

Figura 1. Algoritmo diagnóstico de MS. (Basado en referencias 3 y 7 ). 
IL-6, histamina y heparina los que determinan aumento del remodelamiento óseo a través de su acción sobre sus principales vías de regulación (RANKL/OPG/RANK y Wnt) ${ }^{3,9,10}$. Esta hipótesis se sustenta en estudios que demuestran aumento de marcadores de resorción (CTx y deoxipiridinolina) y formación ósea (fosfatasas alcalinas especificas) en pacientes con MS comparado con controles $\operatorname{sanos}^{3,5}$. En la mayoría de los pacientes esto determina pérdida de DMO y cambios de la microarquitectura que explican el aumento del riesgo de fracturas.

En la literatura se han publicado varias evaluaciones de DMO a través de DXA en pacientes con MS, que muestran que entre 18 al 31\% tendrán osteoporosis. Más aún, al buscar dirigidamente la presencia de fracturas radiográficas vertebrales se describe una prevalencia de alrededor de $20 \%$ en hombres y $14 \%$ en mujeres ${ }^{3,6}$. Esto pone en evidencia la necesidad de realizar estudio dirigido con DXA en todo paciente en que se diagnostica una MS, y en caso de estar alterada, completar el estudio con una radiografía de columna dorso-lumbar dado la alta prevalencia de fracturas asintomáticas ${ }^{3}$. Cabe destacar que el riesgo de compromiso óseo fue similar en los pacientes con o sin compromiso cutáneo, por lo que la única manifestación clínica de una MS pudiese ser la osteoporosis. Esto queda más claramente demostrado en un estudio holandés en que se evaluó a 42 hombres con Osteoporosis Idiopática, dado estudio negativo de causas secundarias, en los cuales se buscó dirigidamente MS con marcadores de actividad mastocitaria y biopsia de MO. En 4 de $42(9 \%)$ esta búsqueda fue compatible con $\mathrm{MS}^{11}$. Estos antecedentes fundamentan, como ilustra también el segundo caso reportado, la importancia de descartar MS en el estudio de pacientes con osteoporosis así llamada Idiopática. Para este efecto, la triptasa sérica es el método de tamizaje de elección ${ }^{3,8}$.

Escasa evidencia ha sido publicada a la fecha respecto al mejor tratamiento de osteoporosis por $\mathrm{MS}^{12,13}$. Dado que se trata de una patología de alto remodelamiento óseo, los antiresortivos son la primera línea de tratamiento. Se han publicado series pequeñas de casos de pacientes tratados con bisfosfonatos que han demostrado aumento significativo de DMO en columna lumbar con efecto limitado en cuello femoral ${ }^{14,15}$. El beneficio en disminución de riesgo de fracturas no ha sido aún demostrado, como tampoco el beneficio de Denosumab, otro antiresortivo recientemente introducido en clínica. De esta forma, dado la ausencia de evidencia respecto a la eficacia y seguridad de estos tratamiento en MS, la indicación de éstos debe ser individualizada caso a caso e idealmente indicada por especialistas.

\section{Conclusión}

En suma, en todo paciente con MS es fundamental descartar la presencia de osteoporosis secundaria. Del mismo modo, al enfrentarse a un paciente con osteoporosis sin causa aparente, debe descartarse la MS como la etiología subyacente. La medición de triptasa sérica es el examen de tamizaje más adecuado para este fin.

\section{Referencias}

1. Cem A, Valent P. Diagnostic Criteria and Classification of Mastocytosis in 2014. Inmunol Allergy Clin N Am 2014; 34: 207-18.

2. Bibi S, Langenfeld F, Jeanningros S, Brenet F, Soucie E, Hermine F, et al. Molecular Defects in Mastocytosis. Kit and beyond Kit. Immunol Allergy Clin N Am 2014; 34 : 239-62.

3. Rossini M, Zanotti R, Viapiana O, Tripi G, Orsolini G, Idolazzi L, et al. Bone Involvement and Osteoporosis in Mastocytocis. Immunol Allergy Clin N Am 2014; 34 : 383-96.

4. Barete S, Assous N, de Gennes C, Grandpeix C, Palmerini F, Dubreuil $\mathrm{P}$, et al. Systemic mastocytosis and bone involvement in a cohort of 75 patients. Ann Rheum Dis 2010; 69: 1838-41.

5. Rossini M, Zanotti R, Bonadonna P, Artuso A, Caruso $\mathrm{B}$, Schena $\mathrm{D}$, et al. Bone mineral density, bone turnover markers and fractures in patients with indolent systemic mastocytosis. Bone 2011; 49 (4): 880-5.

6. Van der Veer E, Van Der Goot W, de Monchy JG, Kluin-Nelemans HC, Van Doormal JJ. High prevalence of fractures and osteoporosis in patients with indolent systemic mastocytosis. Allergy 2012; 67 (3): 431-8.

7. Horny HP, Metcalf DD, Bennett JM, et al. Mastocytosis. En: Swerdlow SH, Campo E, Harris NL, et al. Editores, WHO classification of tumors of hematopoietic and lymphoid tissues; Lyon IARC Press. 2008. p. 54.

8. Schwartz L. Clinical utility of tryptase levels in systemic mastocytosis and associated hematologic disorders. 
Leukemia Research 2001; 25: 553-62.

9. Theoharides T, Bouchera W, Spear K. Serum Interleukin-6 Reflects Disease Severity and Osteoporosis in Mastocytosis Patients. Int Arch Allergy Immunol 2002; 128: 344-50.

10. Guillaume N, Desoutter J, Chandesris O, Merlusca L, Henry I, Georgin-Lavialle S, et al. Bone complications of mastocytosis: a link between clinical and biological characteristics. Am J Med 2013; 126 (1): 75. e1-7.

11. Brumsen C, Papapoulos SE, Lentjes EGWM, Kluin PM, Hamdy NAT. Potential Role for the Mast Cell in the Pathogenesis of Idiopathic Osteoporosis in Men. Bone 2002; 31 (5): 556-61.

12. Arock M, Valent P. Pathogenesis, classification and treatment of mastocytosis: state of the art in 2010 and future perspectives. Expert Rev Hematol 2010; 3 (4): 497-516.

13. Pardanani A, Tefferi A. Systemic mastocytosis in adults: a review on prognosis and treatment based on 342 Mayo Clinic patients and current literature. Curr Opin Hematol 2010; 17 (2): 125-32.

14. Brumsen C, Hamdy NA, Papapoulos SE. Osteoporosis and bone marrow mastocytosis: dissociation of skeletal responses and mast cell activity during long term bisphosphonate therapy. J Bone Miner Res 2002; 17 (4): 567-9.

15. Marshall A, Kavanagh RT, Crisp AJ. The effect of pamidronate on lumbar spine bone density and pain in osteoporosis secondary to systemic mastocytosis. $\mathrm{Br} \mathrm{J}$ Rheumatol 1997; 36 (3): 393-6. 